\title{
Species and genotype diversity of Plasmodium in malaria patients from Gabon analysed by next generation sequencing
}

\author{
Albert Lalremruata ${ }^{1,2+}$, Sankarganesh Jeyaraj ${ }^{1,3 \dagger}$, Thomas Engleitner ${ }^{1,4}$, Fanny Joanny ${ }^{1}$, Annika Lang ${ }^{1}$, \\ Sabine Bélard ${ }^{5,6}$, Ghyslain Mombo-Ngoma7, Michael Ramharter ${ }^{1,2,7,8}$, Peter G. Kremsner ${ }^{1,2,7}$, \\ Benjamin Mordmüller ${ }^{1,2,7^{*}}$ and Jana Held ${ }^{1,2,7}$
}

\begin{abstract}
Background: Six Plasmodium species are known to naturally infect humans. Mixed species infections occur regularly but morphological discrimination by microscopy is difficult and multiplicity of infection (MOl) can only be evaluated by molecular methods. This study investigated the complexity of Plasmodium infections in patients treated for microscopically detected non-falciparum or mixed species malaria in Gabon.

Methods: Ultra-deep sequencing of nucleus (18S rRNA), mitochondrion, and apicoplast encoded genes was used to evaluate Plasmodium species diversity and MOI in 46 symptomatic Gabonese patients with microscopically diagnosed non-falciparum or mixed species malaria.

Results: Deep sequencing revealed a large complexity of coinfections in patients with uncomplicated malaria, both on species and genotype levels. Mixed infections involved up to four parasite species (Plasmodium falciparum, Plasmodium malariae, Plasmodium ovale curtisi, and P. ovale wallikeri). Multiple genotypes from each species were determined from the asexual 18S rRNA gene. 17 of 46 samples (37\%) harboured multiple genotypes of at least one Plasmodium species. The number of genotypes per sample (MOI) was highest in P. malariae $(n=4)$, followed by P. ovale curtisi $(n=3)$, P. ovale wallikeri $(n=3)$, and P. falciparum $(n=2)$. The highest combined genotype complexity in samples that contained mixed-species infections was seven.
\end{abstract}

Conclusions: Ultra-deep sequencing showed an unexpected breadth of Plasmodium species and within species diversity in clinical samples. MOl of P. ovale curtisi, P. ovale wallikeri and P. malariae infections were higher than anticipated and contribute significantly to the burden of malaria in Gabon.

Keywords: Plasmodium malariae, Plasmodium falciparum, Plasmodium ovale wallikeri, Plasmodium ovale curtisi, Nextgeneration sequencing, Amplicon, Metagenomics

\section{Background}

Malaria in humans is caused by six Plasmodium species: Plasmodium falciparum, Plasmodium vivax, Plasmodium malariae, Plasmodium ovale curtisi, P. ovale wallikeri and Plasmodium knowlesi which, although

\footnotetext{
*Correspondence: benjamin.mordmueller@uni-tuebingen.de ${ }^{\dagger}$ Albert Lalremruata and Sankarganesh Jeyaraj contributed equally to this work

1 Institut für Tropenmedizin, Eberhard Karls Universität Tübingen, Wilhelmstraße 27, 72074 Tübingen, Germany

Full list of author information is available at the end of the article
}

zoonotic, is an important pathogen in humans in several regions of South East Asia [1,2]. Whereas current research is focused on malaria caused by $P$. falciparum and increasingly also $P$. vivax, only relatively little effort has been dedicated to research on the other human malaria species. Infections with these species usually present with low parasitaemia; they can persist for long periods and sometimes remain asymptomatic. Besides mono-infections with one Plasmodium species, mixed infections within one individual occur $[3,4]$ and interaction between concurrent species-although not well 
characterized-may play a role in disease progression and outcome $[5,6]$. In clinical care, species determination is commonly performed by light microscopy, which has limited sensitivity and specificity. Double, triple or even quadruple infections can be detected more reliably by molecular methods. In addition, presence of distinct genotypes (strains) of the same species cannot be discerned microscopically. An important proportion of naturally occurring infections consists of multiple genotypes and "multiplicity of infection" (MOI) refers to the number of different genotypes of one species infecting a single host [7]. For P. falciparum, MOI has been associated with several conditions, including age of the host, clinical severity, and transmission intensity [8]. Determination of within-host diversity may be a useful marker to assess the impact of interventions [9].

Genotyping of polymorphic genes such as merozoite surface proteins (MSPs) and glutamate-rich protein (glurp), by nested-PCR is a gold standard method for assessing MOI in P. falciparum infections. An alternative approach uses next generation sequencing (NGS) technologies, which allow high-resolution analyses of a heterogeneous mixture of the parasites within the host [10]. Compared to the standard method, NGS revealed an up to six times higher MOI of P. falciparum in a previous study [11]. By generating multiple reads per sample (usually between 100 and 10,000), this technique is very sensitive and able to detect minor alleles. Recent analysis of the conserved cytochrome b gene ( $c y t b)$ using NGS, detected multiple genotypes infections (up to 4) including non-falciparum species in $10 \%$ of 437 samples collected in Cameroon [12].

Here, a metagenomics sequencing based approach was applied using three pan-Plasmodium primer sets for nucleus (18S rRNA), mitochondrion (cytb), and apicoplast $(\operatorname{clp} C)$ encoded genes to characterize the different Plasmodium species that infect patients with microscopically diagnosed non-falciparum malaria in the department of Tsamba-Magotsi, Gabon, a remote area of rural Central Africa [13]. By this approach, parasite diversity in 46 Gabonese symptomatic patients is described, that are sampled over a time period of 3 years and provide a baseline for research on the epidemiology and potential influence of non-falciparum malaria on disease burden in Central Africa.

\section{Methods}

\section{Patients}

Parasite DNA for this study was extracted from dried blood spots on filter paper of 46 patients with uncomplicated, microscopically diagnosed non-falciparum or mixed species malaria, which represents a minority of malaria cases in the region but was found in more than
$20 \%$ in a cross-sectional survey in the area [13]. Blood samples from two clinical studies were used. From the first study (recruited 2008-2010) samples of 30 patients were included, details of patients' characteristics are published elsewhere [14]. Briefly, patients with uncomplicated malaria, defined as symptoms and presence of $P$. ovale or $P$. malariae in thick blood smear, either as mono or mixed infection were included after informed consent was given. All patients received artemether-lumefantrine as anti-malarial chemotherapy and recovered from the infection. From the second study (recruited 2012-2013) 16 samples of patients that were included for molecular assessment of Plasmodium spp. were used. Both studies were approved by the regional ethics committee (Comité d'Ethique Régional Indépendant de Lambaréné) and followed the principles of the Declaration of Helsinki in its 5 th revision.

\section{Amplification and $\mathbf{4 5 4}$ sequencing}

Capillary blood collected on filter paper from patients prior to anti-malarial treatment was processed for DNA extraction using QIAamp DNA blood mini kit (Qiagen) according to the manufacturer's specifications. For malaria species and genotype identification using NGS, we designed three Plasmodium genus-specific primer sets from the conserved region flanking the highly polymorphic nucleotide sequence of the $18 \mathrm{~S}$ rRNA, the mitochondrial cytochrome $\mathrm{b}(c y t b)$ and the apicoplast caseinolytic protease $\mathrm{C}$ gene $(\operatorname{clpC})$. All primers were 5 -fused to universal tail sequences. Using $2.5 \mu \mathrm{l}$ of DNA extract, target specific PCR was carried out by using Phusion High-Fidelity PCR master mix (Finnzymes) for 35 cycles. The 454 MID kit (Multiplicom) was used to perform subsequent PCR addition of a 454-adaptor sequence linked to multiplex identifiers (MID) in order to discriminate the patients in following analysis steps. Here, the 100 times diluted first round PCR product was used as template and amplified for 20 cycles. All reactions were carried out using a Biometra T2 professional Thermocycler. The corresponding primer sequences and annealing temperature are given in Table 1. PCR amplicons were purified using AmPure XP kit (Agencourt) according to standard procedures (Roche Technical Bulletin No. 2011-007). Quality and purity of amplicons were checked using the Agilent DNA 1000 assay kit on a 2100 Bioanalyzer (Agilent Technology) and subsequently quantified using the Quant-iT Picogreen dsDNA reagent (Invitrogen) on a Fluoroskan Ascent microplate Fluorometer (ThermoScientific). Based on individual DNA concentration, each amplicon was diluted to $10^{7}$ molecules/ $\mu$ l stock solutions. Amplicons were pooled in equimolar concentration to generate a single library and further processed following the GS Junior emPCR LibA 
Table 1 Primers used in this study

\begin{tabular}{lll}
\hline Primer name & $\mathbf{5}^{\prime} \mathbf{- 3}^{\prime}$ sequence $^{\mathbf{a}}$ & Annealing $\left.\mathbf{(}^{\circ} \mathbf{C}\right)$ \\
\hline $\begin{array}{l}\text { SSU-Fwd } \\
\text { SSU-Rev }\end{array}$ & AAGACTCGGCAGCATCTCCAGTGAATTCTTAGATTTTCTG \\
Cyt-Fwd & GCGATCGTCACTGTTCTCCACGTGTTGGTCAAATTAAGC \\
Cyt-Rev & AAGACTCGGCAGCATCTCCAGAGTGGATGGTGTTTAGAT & 58 \\
Clpc-Fwd & GCGATCGTCACTGTTCTCCAGTGCTACCATGTAAATGTAA & 58 \\
Clpc-Rev & AAGACTCGGCAGCATCTCCAGGTCAATTAACAGAACAA & 55 \\
rPLU6 & GCGATCGTCACTGTTCTCCATAGTTAATCTATTTAATAATTC \\
rPLU5 & TTAAATTGTTGCAGTTAAAACG \\
p-AVL-F & CCTGTTGTTGCCTTAAACTTC \\
P-AVL-R & GGAATGACAATGTCGTAAAACAAAGTAT \\
\hline
\end{tabular}

a Underlined region represents universal tag sequences

b Snounou et al. [25]

method (Version April 2011) for emulsion PCR (emPCR) using a low copy per bead ratio $(0.25 \mathrm{cpb}) .500,000 \mathrm{DNA}$ enriched beads were loaded onto a GS Junior Picotiter plate following the GS Junior sequencing manual (Version April 2011) and sequencing was performed in both, forward and reverse direction using the GS Junior Titanium sequencing kit.

\section{Sequence analysis}

Figure 1 summarizes the bioinformatics pipeline used to process Plasmodium sequence reads generated by GS Junior. The sfffile program (SFF Tools, Roche) was used to split raw sequence data based on multiplex identifier (MID). Low quality and short reads $(<200 \mathrm{bp})$ were excluded prior to analysis. Sequencing errors (PCR noise) and homopolymer stretches were corrected with Acacia [15]. Chimeric sequences were detected by using the software Uchime [16] and excluded from further analysis. Both programs were run with default parameters. High-quality filtered reads were mapped to a local reference database comprising $18 \mathrm{~S}$ rRNA, $c y t b$ and $c l p C$ gene sequences of Plasmodium spp. downloaded from GenBank (Table 2). Unmapped sequences were further analyzed using BLAST searches against the NCBI nucleotide database and the Plasmodium database (Plasmodb) [17]. Single nucleotide polymorphisms (SNPs) in the analyzed genes were determined using the probabilistic variant detection method-implemented in the CLC Genomics Workbench 5 (CLC Bio, Aarhus, Denmark). Haplotypes were determined with DnaSP [18] using the following thresholds: SNP frequency equal or greater than $10 \%$ as well as per gene read coverage $\geq 10$ fold. Plasmodium species genotypes were identified by querying consensus sequences against the NCBI GenBank database. All SNP positions were reported as absolute positions in the best matching NCBI reference sequence. Polyclonal infections were identified based on SNPs in the variable region (V5) of the $18 \mathrm{~S}$ gene, giving the number of genotypes infecting one host (referred to as MOI). The number of genotypes obtained by this marker typically leads to an underestimation of polyclonality and characterization of genotypes based on more polymorphic genes might better estimate the scale of multiple infections. However, this would make comparisons between species more difficult as different genes would be evaluated.

\section{Results}

Sequence statistics from patients

The 46 patient samples were analyzed in batches of four sequencing runs on a Roche 454 GS Junior sequencer. The median number of raw reads assigned to each sample was 3829 (range 1563-11,590). However, after removal of chimeric and low-quality reads, the final informative sequences available for each sample was 3165 (range 655-9091). The sequence coverage for each locus per sample is reported in the Additional file 1. Differences in coverage between loci and overall batch to batch variation in the number of raw reads were observed. However, there is no evidence that this substantially influences the results, as the overall coverage per sample is expected to be high enough to detect minor genotypes.

\section{Species discrimination by deep sequencing}

Each filtered read was mapped to the target genes of the reference species for identification. Mixed-species malaria infections identified by the metagenomics approach were also detected by conventional nested-PCR in combination with gel electrophoresis [14]. By NGS, it was possible to achieve a more detailed resolution of the species diversity that included identification of nine novel genotypes and polyclonal infections using the same gene in all six investigated Plasmodium spp. 


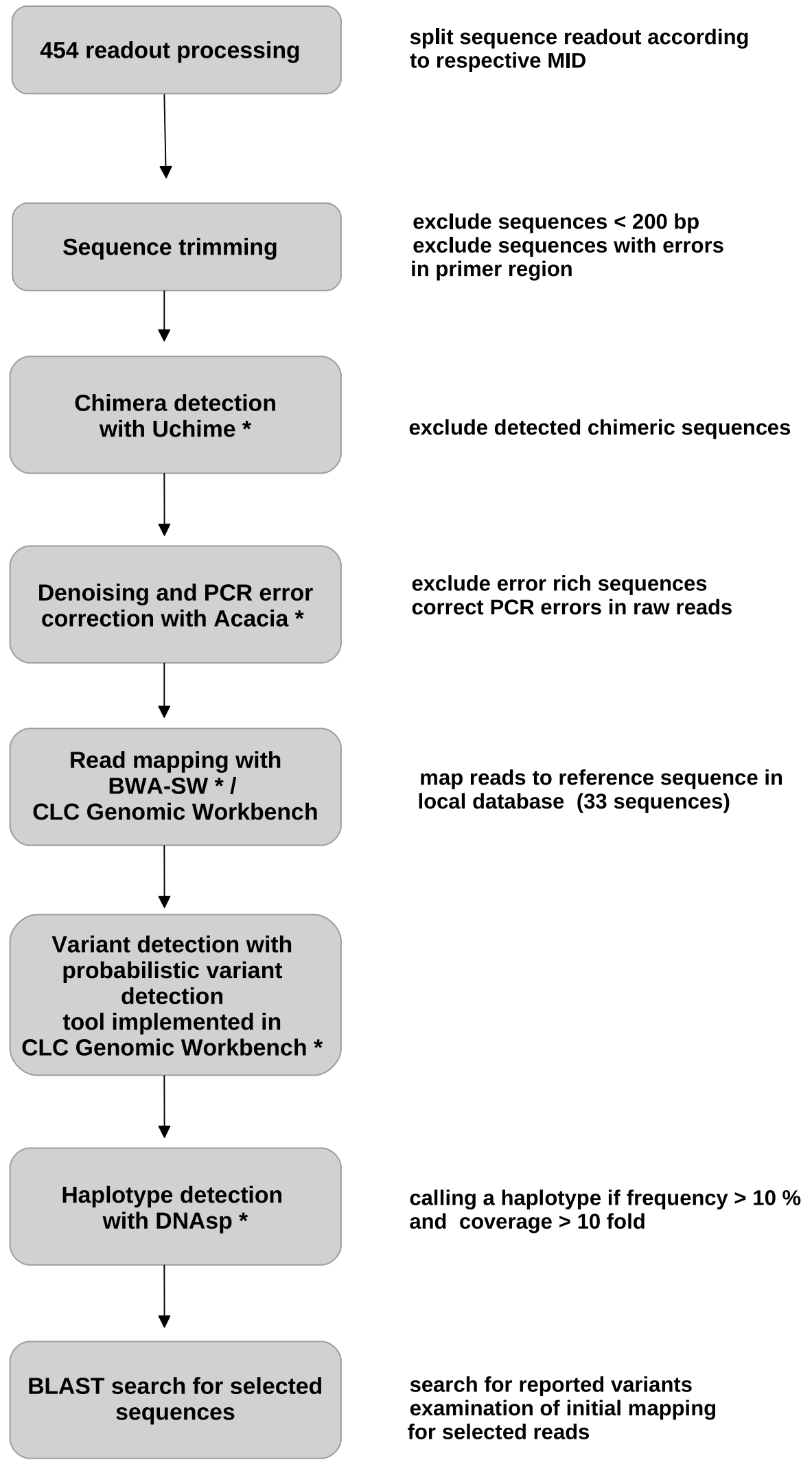

Fig. 1 Bioinformatics pipeline. Flowchart describing bioinformatic pipeline. The pipeline is divided into a quality control part, mapping and variant calling part. * used with default settings 
A minimum of three high quality reads had to be assigned to at least one of the target loci to be included in the analysis. There were $44(96 \%)$ patients with $P$. falciparum, 15 (33\%) with P. malariae, 7 (15\%) with P. ovale curtisi, and $8(17 \%)$ with $P$. ovale wallikeri infections. Different parasite combinations in mixed infections and up to four species in the same host were detected. Of 46 samples, 24 contained only P. falciparum, another two contained only $P$. malariae or $P$. ovale wallikeri. Table 2 Plasmodium spp. reference sequences used
for 454-reads mapping

\begin{tabular}{|c|}
\hline Plasmodium species \\
\hline Plasmodium falciparum $18 \mathrm{~S}$ ribosomal RNA gene (S-type) \\
\hline Plasmodium falciparum $18 \mathrm{~S}$ ribosomal RNA gene (A-type) \\
\hline Plasmodium vivax 185 ribosomal RNA gene (A-Type) \\
\hline Plasmodium vivax 185 ribosomal RNA gene (O-Type) \\
\hline Plasmodium vivax 18 S ribosomal RNA gene (S-Type) \\
\hline Plasmodium berghei $18 \mathrm{~S}$ ribosomal RNA gene \\
\hline Plasmodium berghei $18 \mathrm{~S}$ ribosomal RNA gene \\
\hline Plasmodium malariae $18 \mathrm{~S}$ ribosomal RNA gene \\
\hline Plasmodium ovale $18 \mathrm{~S}$ ribosomal RNA gene \\
\hline Plasmodium ovale $18 \mathrm{~S}$ ribosomal RNA gene \\
\hline Plasmodium yoelii $18 \mathrm{~S}$ ribosomal RNA gene \\
\hline Plasmodium knowlesi $18 \mathrm{~S}$ ribosomal RNA gene \\
\hline Plasmodium cf. inui $18 \mathrm{~S}$ ribosomal RNA gene \\
\hline Plasmodium simiovale $18 \mathrm{~S}$ ribosomal RNA gene \\
\hline Plasmodium ovale wallikeri cytochrome b gene \\
\hline Plasmodium ovale curtisi cytochrome b gene \\
\hline Plasmodium malariae cytochrome b gene \\
\hline Plasmodium vivax cytochrome b gene \\
\hline Plasmodium falciparum cytochrome b gene \\
\hline Plasmodium berghei cytochrome b gene \\
\hline Plasmodium knowlesi cytochrome b gene \\
\hline Plasmodium spp. from Gabon cytochrome b gene \\
\hline Plasmodium yoelii cytochrome b gene \\
\hline $\begin{array}{l}\text { Plasmodium falciparum HB3 clpC gene for caseinolytic } \\
\text { protease } C\end{array}$ \\
\hline $\begin{array}{l}\text { Plasmodium ovale curtisi clpC gene for caseinolytic } \\
\text { protease C }\end{array}$ \\
\hline $\begin{array}{l}\text { Plasmodium ovale wallikeri clpC gene for caseinolytic } \\
\text { protease C }\end{array}$ \\
\hline $\begin{array}{l}\text { Plasmodium malariae clpC gene for caseinolytic protease } \\
\mathrm{C}\end{array}$ \\
\hline Plasmodium vivax clpC gene for caseinolytic protease $\mathrm{C}$ \\
\hline Plasmodium gaboni clpC gene for caseinolytic protease $\mathrm{C}$ \\
\hline Plasmodium knowlesi clpCgene for caseinolytic protease C \\
\hline Plasmodium simiovale clpCgene for caseinolytic protease $C$ \\
\hline Plasmodium cf. ovale clpC gene for caseinolytic protease C \\
\hline Plasmodium yoelii clpC gene for caseinolytic protease $C$ \\
\hline Plasmodium berghei clpC gene for caseinolytic protease C \\
\hline
\end{tabular}
GenBank Acc.

HQ283222.1 JQ627152.1 JQ627158.1 U93235.1 U93234.1 M19712.1 M14599.1 M54897.1 AB182493.1 AB182489.1 AF180727 JF714686.1 FJ619091.1 AB287287.1 KJ930413.1 KP050432.1 LT594637

JN788776

KC175316.1

DQ414645.1

JQ345523.1

AF069623.1

DQ414658.1

DQ642846.1

KP050446.1

KP050439

AB649418.1

AB471871.1

HQ842630.1

AB471880.1

AB471881.1

HQ842632.1

DQ417625.1

AB649421.1
The remaining samples contained double $(\mathrm{n}=15)$, triple $(\mathrm{n}=2)$, and quadruple $(\mathrm{n}=3)$ Plasmodium species infections (Table 3).

\section{Plasmodium falciparum infections}

Plasmodium falciparum specific sequence reads were identified in all but 2 (96\%) of the study samples. Based on the 18S A-type gene sequence, four novel genotypes (GenBank KJ170098-KJ170101) were identified, all are a single nucleotide polymorphism when compared to the reference sequence (GenBank JF681166) as shown in Table 4. No other sequences with these SNPs were found when a similarity search against GenBank was performed. Co-infections with other species were found in 20 patients (45\%).

\section{Plasmodium malariae infections}

A total of 15 infections were observed with the quartan malaria parasite, one as mono and the remaining ones as co-infection with other species. Based on the $18 \mathrm{~S}$ rRNA gene, the parasites were further characterized as $P$. malariae-Asian type $1(\mathrm{n}=1)$ and $P$. malariae-Asian type $2(\mathrm{n}=14)$ [19]. Two unique genotypes (GenBank KJ170105, KJ170106) similar to Plasmodium cf. malariae type 2 were obtained (GenBank AF488000, 99\% identity). Each genotype is defined by either a single nucleotide substitution or insertion compared to $P$. malariae-Asian type 2 (Table 4 ) (see comments below).

\section{Plasmodium ovale wallikeri and $P$. ovale curtisi infections}

Plasmodium ovale wallikeri and P. ovale curtisi were identified in the dataset supported by reads matching to specific $18 \mathrm{~S}, c y t b$, or $c l p C$ sequences of the two $P$. ovale species (Additional file 1). In addition, mixed infections of $P$. ovale curtisi and $P$. ovale wallikeri were observed in four patients (Table 3 ).

Table 3 Number of Plasmodium spp. infections detected by deep sequencing

\begin{tabular}{llc}
\hline Species & Infection type & Patients (n) \\
\hline Pfal & Mono infection & 24 \\
Pmal & Mono infection & 1 \\
Pow & Mono infection & 1 \\
Pfal, Pmal & Double infection & 10 \\
Pfal, Poc & Double infection & 3 \\
Pfal, Pow & Double infection & 2 \\
Pfal, Pmal, Pow & Triple infection & 1 \\
Pfal, Poc, Pow & Triple infection & 1 \\
Pfal, Pmal, Poc, Pow & Quadruple infection & 3 \\
\hline
\end{tabular}

Pfal, P. falciparum; Pmal, P. malariae; Poc, P. ovale curtisi; Pow, P. ovale wallikeri 
Table 4 Polymorphic Nucleotide positions compared to GenBank best-hits reference sequences

\begin{tabular}{|c|c|c|c|c|c|c|c|c|}
\hline Genotypes & Origin & 386 & 407 & 504 & 517 & 529 & & \\
\hline P. falciparum (JF681166) & China & G & $\mathrm{T}$ & G & A & C & & \\
\hline P. falciparum (KJ170098) & Gabon & A & C & & - & . & & \\
\hline P. falciparum (KJ170099) & Gabon & & & & & G & & \\
\hline P. falciparum (KJ170100) & Gabon & A & . & . & . & & & \\
\hline \multirow[t]{2}{*}{ P. falciparum (KJ170101) } & Gabon & . & . & A & . & & & \\
\hline & & 1036 & 1189 & & & & & \\
\hline P. malariae (AF488000) & Myanmar & G & - & & & & & \\
\hline P. malariae (KJ170105) & Gabon & . & $\mathrm{T}$ & & & & & \\
\hline \multirow[t]{2}{*}{ P. malariae (KJ170106) } & Gabon & A & $\mathrm{T}$ & & & & & \\
\hline & & 1005 & 1026 & 1109 & 1112 & 1128 & 1131 & 1132 \\
\hline P. ovale wallikeri (AB182493) & Indonesia & A & C & G & G & A & G & A \\
\hline P. ovale wallikeri (KJ170102) & Gabon & & . & . & . & G & & \\
\hline P. ovale wallikeri (KJ170103) & Gabon & G & $\mathrm{T}$ & A & A & & A & G \\
\hline P. ovale wallikeri (KJ170104) & Gabon & $\mathrm{G}$ & $\mathrm{T}$ & & & . & . & . \\
\hline
\end{tabular}

Each dot represents nucleotide similarity with the GenBank reference sequences. Dashes represent deletions. Numbers at the column header represent nucleotide positions corresponding to GenBank reference sequences

All of $P$. ovale curtisi genotypes identified from the samples were similar to previously submitted sequences. Three novel genotypes of $P$. ovale wallikeri were identified based on 18S rRNA gene sequence polymorphisms (GenBank KJ170102-KJ170104). One of them possesses a single nucleotide substitution at position 1128. Genotype 2 and Genotype 3 showed six and two nucleotide substitutions when compared to the corresponding reference sequence (GenBank AB182493), respectively (Table 4).

\section{Detection of $P$. ovale curtisi $18 \mathrm{~S}$ rRNA gene variant}

In three of the samples a fraction of reads (range 10-15 reads) could not be mapped to any reference sequence. A sequence identity search against the NCBI database initially did not show significant similarity with respect to query coverage and maximum identity with any of the Plasmodium spp. 18S sequence. A 1078 bp length sequence was constructed (GenBank KJ170108), spanning the variable regions 4 and 7 along with the 454 amplicon target (V5) by PCR and Sanger sequencing with pan-Plasmodium and specific primers, respectively (Fig. 2). The generated sequence showed $100 \%$ similarity to the draft genome of $P$. ovale (P. ovale Blast Server, Sanger Institute) and a recently discovered $18 \mathrm{~S}$ rRNA gene variant (GenBank KF696378) of P. ovale curtisi [20].

\section{Multiplicity of infection}

An overview of the MOI based on the variable region (V5) of the $18 \mathrm{~S}$ gene for the different Plasmodium species is given in Table 5 . The relative frequency of each genotype per sample based on the number of reads is plotted in Fig. 3. Multiple P. falciparum genotype (MOI) infections were detected in five patients with a maximum of two genotypes per sample. All $P$. falciparum monoinfections carried a single genotype, except one patient (MID15) who carried two genotypes. Multiple genotype infections were found in two of the seven $P$. ovale curtisi infected samples (MOI of 3). Among eight samples infected with $P$. ovale wallikeri, six harboured multiple $P$. ovale wallikeri genotypes (MOI of 2-3). Analysis of the $c y t b$ and $c l p C$ reads showed $100 \%$ similarity to the published reference sequences. Nine samples contained multiple $P$. malariae genotypes (MOI of 2-4). The number of different genotypes per sample was greater for $P$. malariae when compared to the other species, showing up to four genotypes within one sample.

Reads mapping to cytb and $c l p C$ showed 100\% sequence identity to the reference sequences for all species (KC175316 and DQ642846 for P. falciparum; KJ930413 and KP050439 for P. ovale wallikeri; KP050432, KP050446 for P. ovale curtisi; HQ842634 and AB649418 for P. malariae).

\section{Discussion}

Gabon is highly endemic for malaria with perennial transmission of $P$. falciparum and co-endemicity of $P$. malariae and P. ovale [21, 22]. Gabon has a low population density (6.7 persons per square $\mathrm{km}$ in 2015) [23], but population mobility is high, and a large fraction of inhabitants live close to or within non-cultivated areas, that contain diverse and intact wildlife [24]. Originally, the study was designed to assess the efficacy of artemisinin combination therapy (artemether-lumefantrine) in uncomplicated non-falciparum and mixed species 


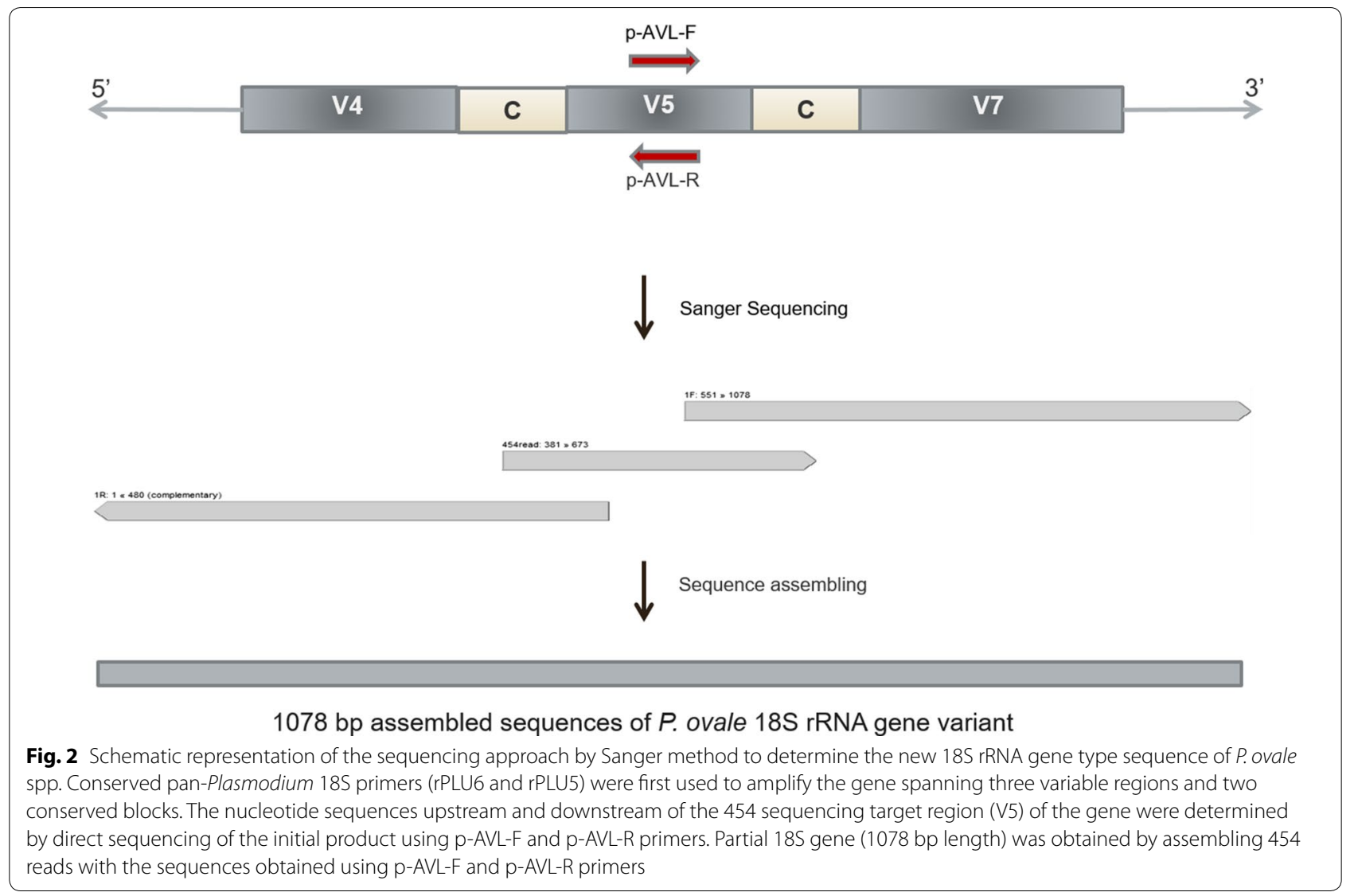

Table 5 Multiplicity of Plasmodium spp. infections

\begin{tabular}{|c|c|c|c|c|c|c|}
\hline \multirow[t]{2}{*}{ Sample id } & \multirow[t]{2}{*}{ Infecting species } & \multicolumn{5}{|c|}{ Number of genotypes for each species } \\
\hline & & Pfal & Pmal & Poc & Pow & Total (MOI) \\
\hline MID01 & Pmal & & 4 & & & 4 \\
\hline MID11 & Pfal, Pmal & 2 & 2 & & & 4 \\
\hline MID15 & Pfal & 2 & & & & 2 \\
\hline MID16 & Pfal, Pmal, Poc, Pow & 1 & 4 & na & 2 & 7 \\
\hline MID23 & Pfal, Poc, Pow & 2 & & & 2 & 4 \\
\hline MID24 & Pfal, Poc & 1 & & 3 & & 4 \\
\hline MID25 & Pfal, Pmal & 1 & 3 & & & 4 \\
\hline MID27 & Pfal, Pmal & 2 & 2 & & & 4 \\
\hline MID28 & Pfal, Pmal, Poc, Pow & 2 & 2 & 1 & 1 & 6 \\
\hline MID30 & Pfal, Pmal, Pow & 1 & na & & 2 & 3 \\
\hline MID31 & Pfal, Poc & 1 & & 3 & & 4 \\
\hline MID32 & Pfal, Pow & 1 & & & 3 & 4 \\
\hline MID34 & Pfal, Pmal & 1 & 2 & & & 3 \\
\hline MID38 & Pfal, Pow & 1 & & & 3 & 4 \\
\hline MID41 & Pfal, Pmal & 1 & 2 & & & 3 \\
\hline MID43 & Pfal, Pmal & 1 & 2 & & & 3 \\
\hline MID46 & Pow & & & & 2 & 2 \\
\hline
\end{tabular}

Pfal, P. falciparum; Pmal, P. malariae; Poc, P. ovale curtisi; Pow, P. ovale wallikeri; na, no $18 \mathrm{~S}$ sequence obtained 


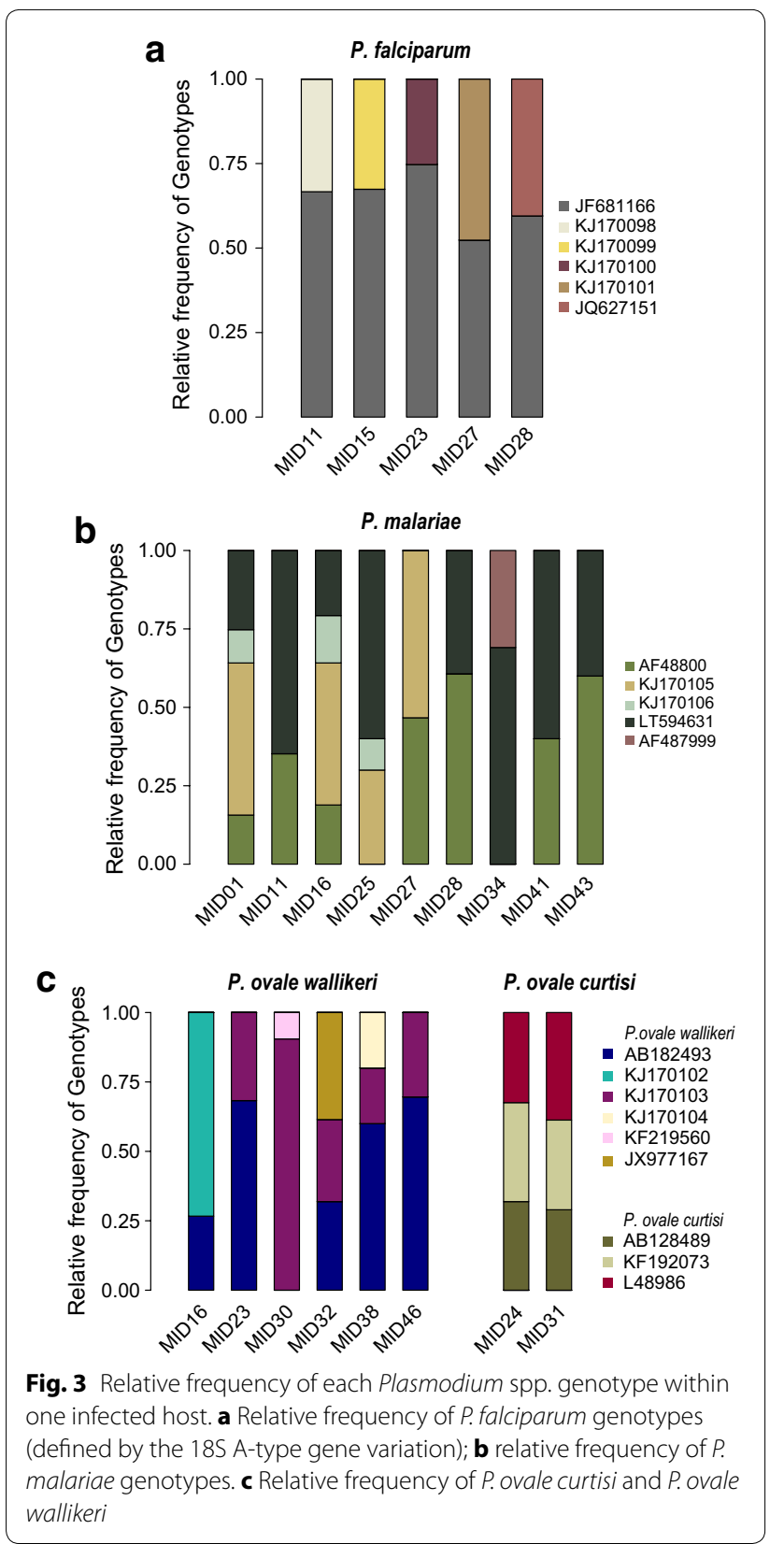

malaria [14]. Species identification was based on microscopy and verified by species-specific nested PCR [25]. Here, a metagenomics approach was designed based on high throughput sequencing to re-analyze the diversity of Plasmodium spp. Two studies were conducted recently using NGS to explore the evidence of zoonotic transmission of Plasmodium spp. in humans [12, 26]. These studies were designed to differentiate Laverania species of apes and humans based on the diagnostic single nucleotide polymorphism found in the mitochondrial DNA (mtDNA). The current study expanded the target genes to three genes including the $18 \mathrm{~S}$ rRNA and $c l p C$ gene to improve accuracy and assess the diversity of Plasmodium populations in blood samples of malaria patients. The $18 \mathrm{~S}$ rRNA gene is one of the most commonly used targets in the molecular diagnosis of Plasmodium spp. including a widely used nested PCR and a number of real-time PCR assays [25, 27]. Due to the high copy numbers (5-10 copies), and the presence of both highly conserved and variable regions, $18 \mathrm{~S}$ genes are well-suited and frequently used molecular targets for the detection and discrimination of Plasmodium species. Until to date, no study has applied these genes to identify Plasmodium spp. using NGS.

As expected, results obtained by conventional speciesspecific PCR were reproduced upon DNA re-extraction and analysis by deep sequencing. Beyond this, this analysis gives a snapshot in time; showing the breadth of co-infections and an unbiased estimate of infection intensity of the different Plasmodium spp. in a cohort of patients with uncomplicated malaria. P. malariae is widespread in Africa but rarely characterized by molecular techniques. Based on morphological differences and sequence polymorphisms compared to genuine $P$. malariae (Uganda CDC isolate), two possible subtypes were identified in Asia: P. cf. malariae type 1 and $P$. cf. malariae type 2 [19]. It would be interesting to investigate if the Asian and the Ugandan type differ in their sensitivity to artemisinins, since a reduced sensitivity of an infection in Uganda has been observed $[28,29]$ but all patients in this study responded well to the treatment [14]. Only few studies have looked at MOI of non-falciparum species. It has been shown in one study that $P$. malariae infections in Malawi, often consisted of multiple genotypes per infected individual and showed a surprisingly similar pattern when compared to $P$. falciparum [30]. Up to five genotypes were detected from a single sample by multilocus genotyping based on microsatellite markers in asymptomatic carriers [31]. There are also up to four different genotypes of $P$. malariae in two patients and three different genotypes of $P$. ovale wallikeri and $P$. ovale curtisi each in two patients in the here presented study based on the $18 \mathrm{~S}$ region, respectively. Many of the $P$. falciparum infections were on the contrary caused only by one genotype when judged by polymorphisms in the $18 \mathrm{~S}$ rRNA gene. These results are surprising as one would assume a lower MOI in P. malariae and both $P$. ovale species as the reported prevalence in this region is low. However, results are also in line with earlier findings from Cambodia for $P$. ovale [32], reflecting the possibility that these parasite species are more prevalent than previously thought. Evidence suggests that $P$. malariae is not completely species-specific and also prevalent in non-human primates [29, 33], having a larger pool of hosts. In addition, infections with these species might be more chronic and long-lived so that multiple genotypes could accumulate in one host. It has to be considered that the marker used 
to define a genotype in this study is not as polymorphic as markers commonly used to define genotypes; for example microsatellites, or genes like $m s p 1, m s p 2$ and glurp for $P$. falciparum [34]. It is expected that the number of genotypes are larger if more polymorphic markers had been used. The advantage of our approach is that homologous genes between the different species can be compared and one can get an impression on the population structure of the co-infecting species. Particularly, because there is no evidence that selection leads to different mutation rates in this gene region for the different species, this can be one approach to compare diversity between species. These results highlight the potential of amplicon-based high throughput sequencing combined with adequate polymorphic markers to obtain reliable molecular characterization of other non-falciparum species, where only limited data are available. Despite short-read length $(<400 \mathrm{nt})$ generated by the NGSs technique, significant dimorphism of the targeted genes allowed accurate assignment of reads between $P$. ovale curtisi and $P$. ovale wallikeri. By using a multiple locus approach, co-infections of the two $P$. ovale species in four patients were detected, adding yet further evidence that the two species do not recombine in nature. The primer for the $c y t b$ gene was selected from a region not including the ape-specific SNPs leading to potential inability to detect non-human species. However, there was no evidence for non-human Plasmodium species infections based on the analysis of the other two genes.

Two structurally distinct types of $18 \mathrm{~S}$ rRNA have been reported in many Plasmodium species [35]. In P. falciparum, type $\mathrm{A}$ and type $\mathrm{S}$ has been described with up to $11 \%$ difference in the sequences between the two types [36]. The existence of paralogous $18 \mathrm{~S}$ rRNA genes in $P$. malariae, $P$. ovale wallikeri and $P$. ovale curtisi genome is not well described until now. The new type of $18 \mathrm{~S}$ rRNA gene sequence from this study, together with the recent report [20], clearly suggests that at least two different forms exist in P. ovale curtisi and P. ovale wallikeri [20]. A real-time PCR based assay targeting this new sequence showed high sensitivity and specificity and can be used for the differential diagnosis of $P$. ovale species infections (unpublished observation).

The main limitation of techniques that involve PCR is the inherent risk of contamination and the NGS approach is no exception. Thus, an essential aspect of sample preparation for sequencing is the careful adoption of experimental strategies aimed at minimizing cross-contamination. In addition, amplicon-based NGS are prone to errors such as chimeras, a well-known issue in metagenomics analyses of environment bacterial communities. Several measures to minimize and avoid these underlying problems at each step, including a non-template control to check contamination of reagents during each target amplification, a separate work station for DNA extraction, PCR assay set-up, and post-PCR processing are necessary.

The true burden of disease caused by $P$. malariae and the $P$. ovale species is not known as these species are mostly underdiagnosed despite their worldwide distribution [29]. Malaria caused by these parasites is generally more benign when compared to $P$. falciparum. Infections present often with a low level parasitaemia that is difficult to detect by microscopy, but might cause a more chronic illness associated with anaemia [37]. Recent reports reveal a higher than expected prevalence of these species in many African countries when diagnosed by PCR [38], going in line with the deep sequencing result. Currently, deep sequencing methods may not be applicable in field settings but can be helpful in longitudinal epidemiological studies to investigate the emergence and change in composition of plasmodial species. The study population was highly selected and represents only a minority of all malaria cases in the area. Microscopic species differentiation is not reliable, particularly when parasite density is low. Therefore, it is unlikely that the appearance of new plasmodial species is noted without molecular techniques. Diversity of the two $P$. ovale species, and characterization of the $P$. malariae population should be taken into consideration for the design, endpoints and feasibility of malaria control strategies, e.g. longer follow up or separate treatment of hypnozoites. Fortunately, artemisinin combination therapy was efficacious in all patients of the present study but emergence of novel zoonotic species or isolates that may be inherently less responsive to current treatment regimens shall be detected early, to provide a specific treatment regimen, especially when severe malaria can occur, as in the case of $P$. knowlesi infections. The impact on malaria vaccine development shall even be stronger since crossprotection between Plasmodium species is limited [39] and mechanisms of high-grade protection are often not known but likely to be different for each parasite species.

\section{Conclusions}

Parasite diversity of Plasmodium species in naturally acquired malaria is larger than expected. The population structure, especially of non-falciparum species, needs further assessments to better understand the prevalence and biology of these parasites. Metagenomics analysis by deep sequencing provides a tool for this and boosts the understanding of naturally acquired malaria. It will be exciting to expand such investigations to other malariaendemic regions and larger cohorts. 


\section{Additional file}

Additional file 1: Table S1. Number of high-quality 454 reads mapped to Plasmodium spp. references.

\begin{abstract}
Abbreviations
clpC: caseinolytic protease C; cytb: cytochrome b; MOl: multiplicity of infection; mtDNA: mitochondrial DNA; NGS: next-generation sequencing; PCR: polymerase chain reaction; SNPs: single nucleotide polymorphisms.
\end{abstract}

\section{Authors' contributions}

$B M, S J$ conceived the idea of the project. SJ, AL, TE, MR, SB, PGK, and BM designed research. $A L$, SJ performed NGS experiments. AL, SJ, TE, JH and BM analysed data and drafted the manuscript. FJ, AL and GMN contributed to data collection. All authors interpreted the data. All authors read and approved the final manuscript.

\section{Author details}

${ }^{1}$ Institut für Tropenmedizin, Eberhard Karls Universität Tübingen, Wilhelmstraße 27, 72074 Tübingen, Germany. ${ }^{2}$ German Centre for Infection Research, partner site Tübingen, Wilhelmstraße 27, 72074 Tübingen, Germany. ${ }^{3}$ PSG Institute of Advanced Studies, Coimbatore 641004 , India. ${ }^{4}$ Department of Medicine II, Klinikum rechts der Isar, Technische Universität München, Munich, Germany. ${ }^{5}$ Department of Pediatric Pneumology and Immunology, Charité-Universitätsmedizin Berlin, Berlin, Germany. ${ }^{6}$ Berlin Institute of Health, Berlin, Germany. ${ }^{7}$ Centre de Recherches Médicales de Lambaréné (CERMEL), Lambaréné, Gabon. ${ }^{8}$ Department of Medicine I, Division of Infectious Diseases and Tropical Medicine, Medical University of Vienna, Vienna, Austria.

\section{Acknowledgements}

We thank all the participants who agreed to participate in this study, the staff of the Institute of Tropical Medicine, Tübingen and Centre de Recherches Médicales de Lambaréné (CERMEL), Lambaréné, Gabon.

\section{Competing interests}

The authors declare that they have no competing interests.

\section{Availability of data and materials}

The datasets used and/or analysed during the current study available from the corresponding author on reasonable request. The new $18 \mathrm{~S}$ genotypes reported in this paper are available in the GenBank under the Accession Number(s): KJ170100.1 to KJ170108.1

\section{Consent for publication}

Not applicable.

\section{Ethics approval and consent to participate}

Both studies were approved by the responsible regional ethics committee, Comité d'Ethique Régional Indépendant de Lambaréné, Gabon. All study participants consented to participate in the study.

\section{Funding}

Part of the study was funded by the German Federal Ministry of Education and Research (01KA1004 and 01KA0803).

\section{Publisher's Note}

Springer Nature remains neutral with regard to jurisdictional claims in published maps and institutional affiliations.

Received: 14 June 2017 Accepted: 22 September 2017 Published online: 03 October 2017

\section{References}

1. Antinori S, Galimberti L, Milazzo L, Corbellino M. Plasmodium knowlesi: the emerging zoonotic malaria parasite. Acta Trop. 2013;125:191-201.
2. Ansari HR, Templeton TJ, Subudhi AK, Ramaprasad A, Tang J, Lu F, et al. Genome-scale comparison of expanded gene families in Plasmodium ovale wallikeri and Plasmodium ovale curtisi with Plasmodium malariae and with other Plasmodium species. Int J Parasitol. 2016;46:685-96.

3. Richie TL. Interactions between malaria parasites infecting the same vertebrate host. Parasitology. 1988;96:607-39.

4. Bruce MC, Donnelly CA, Alpers MP, Galinski MR, Barnwell JW, Walliker D, et al. Cross-species interactions between malaria parasites in humans. Science. 2000;287:845-8.

5. Luxemburger C, Ricci F, Nosten F, Raimond D, Bathet S, White NJ. The epidemiology of severe malaria in an area of low transmission in Thailand. Trans R Soc Trop Med Hyg. 1997;91:256-62.

6. May J, Falusi AG, Mockenhaupt FP, Ademowo OG, Olumese PE, Bienzle $U$, et al. Impact of subpatent multi-species and multi-clonal plasmodial infections on anaemia in children from Nigeria. Trans R Soc Trop Med Hyg. 2000;94:399-403.

7. Smith T, Felger I, Tanner M, Beck HP. Premunition in Plasmodium falciparum infection: insights from the epidemiology of multiple infections. Trans R Soc Trop Med Hyg. 1999;93(Suppl 1):59-64

8. Mayengue PI, Luty AJF, Rogier C, Baragatti M, Kremsner PG, Ntoumi F. The multiplicity of Plasmodium falciparum infections is associated with acquired immunity to asexual blood stage antigens. Microbes Infect. 2009;11:108-14.

9. Juliano JJ, Gadalla N, Sutherland CJ, Meshnick SR. The perils of PCR: can we accurately "correct" antimalarial trials? Trends Parasitol. 2010;26:119-24.

10. Assefa SA, Preston MD, Campino S, Ocholla H, Sutherland CJ, Clark TG. est$\mathrm{MOl}$ : estimating multiplicity of infection using parasite deep sequencing data. Bioinformatics. 2014:30:1292-4.

11. Juliano JJ, Porter K, Mwapasa V, Sem R, Rogers WO, Ariey F, et al. Exposing malaria in-host diversity and estimating population diversity by capturerecapture using massively parallel pyrosequencing. Proc Natl Acad Sci USA. 2010;107:20138-43.

12. Sundararaman SA, Liu W, Keele BF, Learn GH, Bittinger K, Mouacha F, et al. Plasmodium falciparum-like parasites infecting wild apes in southern Cameroon do not represent a recurrent source of human malaria. Proc Natl Acad Sci USA. 2013;110:7020-5.

13. Manego RZ, Mombo-Ngoma G, Witte M, Held J, Gmeiner M, Gebru T, et al. Demography, maternal health and the epidemiology of malaria and other major infectious diseases in the rural department TsambaMagotsi, Ngounie Province, in central African Gabon. BMC Public Health. 2017:17:130.

14. Mombo-Ngoma G, Kleine C, Basra A, Würbel H, Diop DA, Capan M, et al. Prospective evaluation of artemether-lumefantrine for the treatment of non-falciparum and mixed-species malaria in Gabon. Malar J. 2012:11:120.

15. Bragg L, Stone G, Imelfort M, Hugenholtz P, Tyson GW. Fast, accurate error-correction of amplicon pyrosequences using Acacia. Nat Methods. 2012:9:425-6.

16. Edgar RC, Haas BJ, Clemente JC, Quince C, Knight R. UCHIME improves sensitivity and speed of chimera detection. Bioinformatics. 2011;27:2194-200.

17. Aurrecoechea C, Brestelli J, Brunk BP, Dommer J, Fischer S, Gajria B, et al. PlasmoDB: a functional genomic database for malaria parasites. Nucleic Acids Res. 2009:37(Database issue):D539-43.

18. Librado P, Rozas J. DnaSP v5: a software for comprehensive analysis of DNA polymorphism data. Bioinformatics. 2009:25:1451-2.

19. Kawamoto F, Win TT, Mizuno S, Lin K, Kyaw O, Tantulart IS, et al. Unusual Plasmodium malariae-like parasites in southeast Asia. J Parasitol. 2002:88:350-7.

20. Li M, Xia Z, Yan H. New type of SSUrDNA sequence was detected from both Plasmodium ovale curtisi and Plasmodium ovale wallikeri samples. Malar J. 2014;13:216.

21. Wildling $E$, Winkler $S$, Kremsner PG, Brandts $C$, Jenne L, Wernsdorfer WH. Malaria epidemiology in the province of Moyen Ogoov, Gabon. Trop Med Parasitol. 1995:46:77-82.

22. Sylla EH, Kun JF, Kremsner PG. Mosquito distribution and entomological inoculation rates in three malaria-endemic areas in Gabon. Trans R Soc Trop Med Hyg. 2000;94:652-6.

23. World Population Prospects. The 2015 Revision, custom data acquired via website; 2015. 
24. Ramharter M, Adegnika AA, Agnandji ST, Matsiegui PB, Grobusch MP, Winkler $\mathrm{S}$, et al. History and perspectives of medical research at the Albert Schweitzer Hospital in Lambaréné, Gabon. Wien Klin Wochenschr. 2007;119:8-12.

25. Snounou G, Viriyakosol S, Zhu XP, Jarra W, Pinheiro L, do Rosario VE, et al. High sensitivity of detection of human malaria parasites by the use of nested polymerase chain reaction. Mol Biochem Parasitol. 1993;61:315-20.

26. Délicat-Loembet L, Rougeron V, Ollomo B, Arnathau C, Roche B, Elguero $E$, et al. No evidence for ape Plasmodium infections in humans in Gabon. PLOS ONE. 2015:10:e126933.

27. Murphy SC, Hermsen CC, Douglas AD, Edwards NJ, Petersen I, Fahle GA, et al. External quality assurance of malaria nucleic acid testing for clinical trials and eradication surveillance. PLoS ONE. 2014;9:e97398.

28. Calleri G, Balbiano R, Caramello P. Are artemisinin-based combination therapies effective against Plasmodium malariae? J Antimicrob Chemother. 2013;68:1447-8.

29. Rutledge GG, Böhme U, Sanders M, Reid AJ, Cotton JA, Maiga-Ascofare $\mathrm{O}$, et al. Plasmodium malariae and $P$. ovale genomes provide insights into malaria parasite evolution. Nature. 2017;542:101-4.

30. Bruce MC, Macheso A, McConnachie A, Molyneux ME. Comparative population structure of Plasmodium malariae and Plasmodium falciparum under different transmission settings in Malawi. Malar J. 2011;10:38.

31. Bruce MC, Macheso A, Galinski MR, Barnwell JW. Characterization and application of multiple genetic markers for Plasmodium malariae. Parasitology. 2007;134:637-50.
32. Incardona S, Chy S, Chiv L, Nhem S, Sem R, Hewitt S, et al. Large sequence heterogeneity of the small subunit ribosomal RNA gene of Plasmodium ovale in Cambodia. Am J Trop Med Hyg. 2005;72:719-24.

33. Lalremruata A, Magris M, Vivas-Martínez S, Koehler M, Esen M, Kempaiah $P$, et al. Natural infection of Plasmodium brasilianum in humans: man and monkey share quartan malaria parasites in the Venezuelan Amazon. EBioMedicine. 2015;2:1186-92.

34. WHO. Methods and techniques for clinical trials on antimalarial drug efficacy: genotyping to identify parasite populations: informal consultation organized by the medicines for malaria venture and cosponsored by the World Health Organization, 29-31 May 2007. Amsterdam: WHO; 2008.

35. Rooney AP. Mechanisms underlying the evolution and maintenance of functionally heterogeneous $18 \mathrm{~S}$ rRNA genes in Apicomplexans. Mol Biol Evol. 2004;21:1704-11.

36. McCutchan TF, de la Cruz VF, Lal AA, Gunderson JH, Elwood HJ, Sogin ML. Primary sequences of two small subunit ribosomal RNA genes from Plasmodium falciparum. Mol Biochem Parasitol. 1988;28:63-8.

37. Langford S, Douglas NM, Lampah DA, Simpson JA, Kenangalem E, Sugiarto $\mathrm{P}$, et al. Plasmodium malariae infection associated with a high burden of anemia: a hospital-based surveillance study. PLOS NeglTrop Dis. 2015;9:e0004195.

38. Daniels RF, Deme AB, Gomis JF, Dieye B, Durfee K, Thwing Jl, et al. Evidence of non-Plasmodium falciparum malaria infection in Kédougou, Sénégal. Malar J. 2017;16:9.

39. Zimmerman PA, Mehlotra RK, Kasehagen LJ, Kazura JW. Why do we need to know more about mixed Plasmodium species infections in humans? Trends Parasitol. 2004:20:440-7.

\section{Submit your next manuscript to BioMed Central and we will help you at every step:}

- We accept pre-submission inquiries

- Our selector tool helps you to find the most relevant journal

- We provide round the clock customer support

- Convenient online submission

- Thorough peer review

- Inclusion in PubMed and all major indexing services

- Maximum visibility for your research

Submit your manuscript at www.biomedcentral.com/submit
() Biomed Central 